\title{
On Marginal Distributions of the Ordered Eigenvalues of Certain Random Matrices
}

\author{
Haochuan Zhang, ${ }^{1}$ Shi Jin, ${ }^{2}$ Xin Zhang, ${ }^{1}$ and Dacheng Yang ${ }^{1}$ \\ ${ }^{1}$ School of Information and Communication Engineering, Beijing University of Posts and Telecommunications, Beijing 100876, China \\ ${ }^{2}$ National Mobile Communications Research Laboratory, Southeast University, Nanjing 210096, China
}

Correspondence should be addressed to Haochuan Zhang, zhcbupt@gmail.com

Received 27 November 2009; Revised 13 May 2010; Accepted 2 July 2010

Academic Editor: Athanasios Rontogiannis

Copyright ( $\odot 2010$ Haochuan Zhang et al. This is an open access article distributed under the Creative Commons Attribution License, which permits unrestricted use, distribution, and reproduction in any medium, provided the original work is properly cited.

This paper presents a general expression for the marginal distributions of the ordered eigenvalues of certain important random matrices. The expression, given in terms of matrix determinants, is compacter in representation and more efficient in computational complexity than existing results in the literature. As an illustrative application of the new result, we then analyze the performance of the multiple-input multiple-output singular value decomposition system. Analytical expressions for the average symbol error rate and the outage probability are derived, assuming the general double-scattering fading condition.

\section{Introduction}

Random matrix theory, since its inception, has been known as a powerful tool for solving practical problems arising in physics, statistics, and engineering [1-3]. Recently, an important aspect of random matrix theory, that is, the distribution of the eigenvalues of random matrices, has been successfully applied to the analysis and design of wireless communication systems [4]. These applications, mostly concerning the multiple-input multiple-output (MIMO) systems, can be summarized as follows. In single-user MIMO systems, the eigenvalue distributions of Wishart matrices (a Wishart matrix [1] is formed by multiplying a Gaussian random matrix (of the size $m \times n$ ) with its Hermitian transposition (given that $m \leq n$ ). If $m>n$, the product matrix was termed the pseudo-Wishart matrix [5]) were widely applied to the analysis of MIMO channel capacity [6-11] and specific MIMO techniques, such as MIMO maximum ratio combining (MIMO MRC) (MIMO MRC is a technique that transmits signals along the strongest eigendirection of the channel. It was also known as maximumratio transmission [12], transmit-receive diversity [13], and MIMO beamforming [14]) [15-17] and, MIMO singular value decomposition (MIMO SVD) (MIMO SVD, also known as MIMO multichannel beamforming [18], and spatial multiplexing MIMO $[19,20]$, is a generalization of MIMO MRC. It transmits multiple data streams along several strongest eigen-directions of the channel). [18-21], given that the MIMO channel was Rayleigh/Rician faded. For channels that are not Rayleigh/Rician faded (e.g., the double-scattering [22] fading channel to be discussed in Section 4), the eigenvalue distributions of Wishart matrices also played an essential role in the performance analysis of MIMO systems [23-30]. Even for relay channels, statistical distributions of the eigenvalues were shown very useful in the derivation of the channel capacity $[31,32]$. In multiuser MIMO systems, the eigenvalue distribution of a random matrix (characterized by the channel matrix of the desired user and that of the interferers) was applied to the performance analysis of MIMO optimum combing (MIMO OC) [33-41]. Furthermore, in cognitive radio networks, the eigenvalue distributions of random matrices were recently applied to devise effective algorithms for spectrum sensing [42-44].

Given its importance in various applications, the eigenvalue distribution of random matrices is arguably one of the hottest topic in communication engineering. During the past two years, general methods for obtaining these eigenvalue distributions were developed, applying for a general class of random matrices. To be specific, Ordóñez et al. [20] 
presented a general expression for the marginal distributions of the ordered eigenvalues, while Zanella et al. [45, 46] proposed alternative expressions for the same distributions. The results, however, need separate expressions to cover the Wishart and pseudo-Wishart matrices. This problem was later avoided in the new expression of Chiani and Zanella [21], which was given in terms of the "determinant" of the rank-3 tensor. After that, a simpler expression for the eigenvalue distribution was presented by Sun et al. [41], where only conventional (2-dimensional) determinants were involved.

In this paper, we aim at finding a new expression for the eigenvalue distribution, which is even simpler than Sun's result. To that end, we first show that many important random matrices, especially those in the summary above, share a common structure on the joint distributions of their (nonzero) eigenvalues. Based on the common structure, we then derive the marginal distributions of the ordered eigenvalues, using a classical result from the theory of order statistics, along with the multilinear property of the determinant. It turns out that the new expression we obtained is compacter in representation and more efficient in computational complexity, when comparing with existing results. The new result can unify the eigenvalue distributions of Wishart and pseudo-Wishart matrices with only a single expression. Moreover, it is given in conventional (2-dimensional) determinants, and importantly, it replaces many functions in Sun's result with constant numbers, greatly improving the computational efficiency. As an illustrative application of the new expression, we analyze the performance of MIMO SVD systems, assuming the (uncorrelated) double-scattering [22] fading channels. It is worth noting that, different from the Rayleigh/Rician fading channels, where the performance of MIMO SVD were well-studied in [18], the behaviors of MIMO SVD in double-scattering channels is still not clear (expect for some primary results in [47] by the authors). In this context, we derive first the joint eigenvalue distribution of the MIMO channel matrix, using the law of total probability. Then, based on the joint distribution, we apply the general result to get the marginal distribution for each ordered eigenvalue. After that, we analyze the performance of the MIMO SVD. Analytical expressions for the average SER and the outage probability of the system are derived and validated (with numerical simulations). As the simulation results illustrate, the analytical expressions agree perfectly with the Monte Carlo results.

The rest of this paper is organized as follows. Section 2 presents the common structure of the joint eigenvalue distributions. Based on the common structure, Section 3 derives the general expression for the marginal eigenvalue distributions. Then, in Section 4, we analyze the performance of the MIMO SVD in double-scattering channels, by applying the general result. Finally, we summarize the paper in Section 5. Next, we list the notations used throughout this paper: all vectors and matrices are represented with bold symbols; ${ }^{T}$ denotes the transposition of a matrix; ${ }^{H}$ denotes the Hermitian transposition of a matrix; $0_{m \times n}$ denotes an $m \times n$ matrix with only zero elements; $\mathbf{I}_{m}$ denotes the $m \times m$ identity matrix; $\mathbf{A} \in \mathbb{C}^{m \times n}$ denotes that $\mathbf{A}$ is an $m \times n$ complex matrix; $\{\mathbf{A}\}_{i, j}$ is the $(i, j)$ th element of a matrix $\mathrm{A} ;|\cdot|$ denotes the determinant of a matrix; $\left|\left\{a_{i, j}\right\}\right|$ is the determinant of a matrix whose $(i, j)$ th element is $a_{i, j} ; \mathbb{E}_{\xi}(\cdot)$ is the expectation of a random variable with respect to $\xi$; $\mathbf{A} \sim \mathcal{C} \mathcal{N}_{m \times n}(\mathbf{M}, \boldsymbol{\Omega}, \boldsymbol{\Sigma})$ denotes that $\mathbf{A}$ is an $m \times n$ complex Gaussian matrix with a mean value $\mathbf{M} \in \mathbb{C}^{m \times n}$, a row correlation $\boldsymbol{\Omega} \in \mathbb{C}^{m \times m}$, and a column correlation $\boldsymbol{\Sigma} \in \mathbb{C}^{n \times n}$.

\section{Joint Distributions of Ordered Eigenvalues}

In this section, we show that the random matrices discussed in Section 1 share a common structure on the joint probability density functions (PDFs) of their eigenvalues. (Although the common structure can be found in various random matrices (Rayleigh, Rician, and double-scattering, etc.), it is not true that all random matrices have this structure on the joint PDF of their eigenvalues. A good example in this point is the Nakagami-Hoyt channel, whose joint eigenvalue PDF of the channel matrix is different from (1), see [48, Equation (10)], for more details. It is also worth noting that, for non-Gaussian random matrices, obtaining exact expressions on their joint eigenvalue distributions is generally difficult. Very few results can be found in the literature. In this paper, we focus on exact eigenvalue distributions, and thus, we consider mainly Gaussian and Gaussian-related random matrices.) Indeed, this common structure (formulated as the proposition below) was previously reported in $[20,45,49]$ among others.

Proposition 1. Let $\mathbf{W}$ denote a Hermitian random matrix discussed in Section 1, and let $\lambda=\left(\lambda_{1}, \lambda_{2}, \ldots, \lambda_{m}\right), b \geq \lambda_{1} \geq$ $\lambda_{2} \geq \cdots \geq \lambda_{m} \geq a$, denote the nonzero ordered eigenvalues of $\mathbf{W}$. Then, the joint PDF of $\boldsymbol{\lambda}$ can be expressed as

$$
\begin{gathered}
f_{\lambda}(\mathbf{x})=K|\boldsymbol{\Phi}(\mathbf{x})||\boldsymbol{\Xi}(\mathbf{x})| \prod_{i=1}^{m} v\left(x_{i}\right), \\
\left(b \geq x_{1} \geq x_{2} \geq \cdots \geq x_{m} \geq a\right),
\end{gathered}
$$

where $\mathbf{x}=\left(x_{1}, \ldots, x_{m}\right), f_{\lambda}(\cdot)$ is the joint PDF of $\lambda, K$ is a constant coefficient, $\nu(\cdot)$ is a generic function, $\Phi(\mathbf{x})$ and $\Psi(\mathbf{x})$ are $n \times n$ and $m \times m$ matrices $(n \geq m)$, respectively, whose elements are given by

$$
\begin{gathered}
\{\boldsymbol{\Phi}(\mathbf{x})\}_{i, j}= \begin{cases}\phi_{i}\left(x_{j}\right), & i=1, \ldots, n, j=1, \ldots, m, \\
\phi_{i, j}, & i=1, \ldots, n, j=m+1, \ldots, n,\end{cases} \\
\{\boldsymbol{\Xi}(\mathbf{x})\}_{i, j}=\xi_{i}\left(x_{j}\right), \quad i, j=1, \ldots, m,
\end{gathered}
$$

with $\phi_{i, j}$ being an arbitrary constant, $\phi_{i}(\cdot)$ 's and $\xi_{i}(\cdot)$ 's being a generic function.

Next, we verify the proposition above with random matrices discussed in Section 1. (Let $\mathbf{G}_{1}$ and $\mathbf{G}_{2}$ denote two mutually independent complex Gaussian matrices). 
(i) Single-user MIMO systems:

(a) (uncorrelated) Rayleigh fading channels: Let $\mathbf{G}_{1} \sim$ $\mathcal{C} \mathcal{N}_{N \times M}\left(0_{N \times M}, \mathbf{I}_{N}, \mathbf{I}_{M}\right)$ with $N \geq M$, then the joint $\mathrm{PDF}$ of the eigenvalues of the Wishart matrix $\mathbf{W}=$ $\mathbf{G}_{1}^{H} \mathbf{G}_{1}$ is [6]

$$
\begin{array}{r}
f_{\lambda}(\mathbf{x})=K|\boldsymbol{\Phi}(\mathbf{x})||\boldsymbol{\Xi}(\mathbf{x})| \prod_{i=1}^{M} x_{i}^{N-M} e^{-x_{i}}, \\
\left(\infty>x_{1} \geq \cdots \geq x_{M} \geq 0\right),
\end{array}
$$

where $\boldsymbol{\lambda}=\left(\lambda_{1}, \ldots, \lambda_{M}\right), \mathbf{x}=\left(x_{1}, \ldots, x_{M}\right)$, and

$$
\begin{gathered}
K=\frac{1}{\prod_{i=1}^{M}(N-i) !(M-i) !}, \\
\{\boldsymbol{\Phi}(\mathbf{x})\}_{i, j}=x_{i}^{j-1}, \quad i, j=1, \ldots, M, \\
\{\boldsymbol{\Xi}(\mathbf{x})\}_{i, j}=x_{i}^{j-1}, \quad i, j=1, \ldots, M .
\end{gathered}
$$

Clearly, the joint PDF above is in the form of (1). For semicorrelated Rayleigh and uncorrelated Rician fading channels, one can also verify that the joint PDFs are in the same form as $(1)$, see $[18,20,45,46]$.

(b) Double-scattering channels: Let $\mathbf{G}_{1} \sim$ $\mathcal{C} \mathcal{N}_{N_{r} \times N_{s}}\left(0_{N_{r} \times N_{s}}, \mathbf{I}_{N_{r}}, \mathbf{I}_{N_{s}}\right)$, and $\mathbf{G}_{2}$ $\mathcal{C} \mathcal{N}_{N_{s} \times N_{t}}\left(0_{N_{s} \times N_{t}}, \mathbf{I}_{N_{s}}, \mathbf{I}_{N_{t}}\right)$, with $N_{r}, N_{s}$, and $N_{t}$ being three natural numbers, then the nonzero ordered eigenvalues of $\mathbf{W}=\mathbf{G}_{2}^{H} \mathbf{G}_{1}^{H} \mathbf{G}_{1} \mathbf{G}_{2} / N_{s}$ are jointly distributed as

$$
\begin{array}{r}
f_{\lambda}(\mathbf{x})=K|\boldsymbol{\Phi}(\mathbf{x})||\boldsymbol{\Xi}(\mathbf{x})| \prod_{i=1}^{M} x_{i}^{N-S}, \\
\left(\infty>x_{1} \geq \cdots \geq x_{M} \geq 0\right),
\end{array}
$$

where $\boldsymbol{\lambda}=\left(\lambda_{1}, \ldots, \lambda_{M}\right), \mathbf{x}=\left(x_{1}, \ldots, x_{M}\right)$, and

$$
\begin{aligned}
S & =\min \left(N_{r}, N_{s}\right), \quad T=\max \left(N_{r}, N_{s}\right), \\
M & =\min \left(S, N_{t}\right), \quad N=\max \left(S, N_{t}\right), \\
K & =\frac{(-1)^{(S-M)(S+M-1) / 2} N_{s}^{S T}}{\prod_{i=1}^{S}(S-i) !(T-i) ! \prod_{i=1}^{M}\left(N_{t}-i\right) !},
\end{aligned}
$$

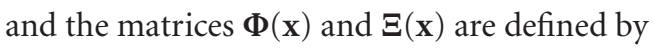

$$
\begin{aligned}
& \{\boldsymbol{\Phi}(\mathbf{x})\}_{i, j} \\
& \left\{\begin{array}{r}
2\left(\frac{x_{j}}{N_{s}}\right)^{\left(T-N_{t}+i-1\right) / 2} \\
K_{T-N_{t}+i-1}\left(2 \sqrt{N_{s} x_{j}}\right), \\
i=1, \ldots, S ; j=1, \ldots, M . \\
(-1)^{S-j}(T-M-N+i+j-2) ! N_{s}^{-(T-M-N+i+j-1)}, \\
i=1, \ldots, S ; j=M+1, \ldots, S . \\
\{\boldsymbol{\Xi}(\mathbf{x})\}_{i, j}=x_{i}^{j-1}, \quad i, j=1, \ldots, M,
\end{array}\right.
\end{aligned}
$$

with $K_{v}(\cdot)$ being the modified Bessel function of the second kind [50, Equation (8.432.6)].

Proof. See Appendix A.

Again, the joint distribution fits well in the from of Proposition 1. More results pertaining to double-scattering channels can be found in [47, Lemma 1].

(ii) Multiuser MIMO systems:

(a) OC without thermal noise: Let $\mathbf{G}_{1} \sim \mathcal{C} \mathcal{N}_{P \times Q}(\mathbf{M}$, $\left.\boldsymbol{\Sigma}, \mathbf{I}_{Q}\right)$ with $Q \geq P, \mathbf{G}_{2} \sim \mathcal{C} \mathcal{N}_{P \times N}\left(0_{P \times N}, \boldsymbol{\Sigma}, \mathbf{I}_{N}\right)$ with $N \geq P$, and $\mathbf{M}^{H} \boldsymbol{\Sigma}^{-1} \mathbf{M}$ has $P$ positive and descendingly ordered eigenvalues $\left(\mu_{1}, \ldots, \mu_{P}\right)$, then the eigenvalues of $\mathbf{W}=\mathbf{G}_{1}^{H}\left(\mathbf{G}_{2} \mathbf{G}_{2}^{H}\right)^{-1} \mathbf{G}_{1}$ are jointly distributed as [38]

$$
f_{\lambda}(\mathbf{x})=K|\boldsymbol{\Phi}(\mathbf{x})||\boldsymbol{\Xi}(\mathbf{x})| \prod_{i=1}^{P} \frac{x_{i}^{Q-P}}{\left(1+x_{i}\right)^{Q+N-P+1}},
$$

$$
\left(\infty>x_{1} \geq \cdots \geq x_{P} \geq 0\right) \text {, }
$$

where $\lambda=\left(\lambda_{1}, \ldots, \lambda_{P}\right), \mathbf{x}=\left(x_{1}, \ldots, x_{P}\right)$, and

$$
\begin{gathered}
K=\frac{e^{-\mu_{i}}}{\prod_{1 \leq i<j \leq P}\left(\mu_{i}-\mu_{j}\right)} \prod_{i=1}^{P} \frac{(N+Q-P) !}{(Q-P) !(N-i) !}, \\
\{\boldsymbol{\Phi}(\mathbf{x})\}_{i, j}={ }_{1} F_{1}\left(Q+N-P+1 ; Q-P+1 ; \frac{x_{i} \mu_{j}}{1+x_{i}}\right), \\
i, j=1, \ldots, P, \\
\{\boldsymbol{\Xi}(\mathbf{x})\}_{i, j}=x_{i}^{j-1}, \quad i, j=1, \ldots, P,
\end{gathered}
$$

with ${ }_{1} F_{1}(\cdot ; \cdot ; \cdot)$ being the generalized hypergeometric function [50, Equation (9.210.1)].

Obviously, the joint PDF here also belongs to the class defined by Proposition 1 . For more examples, see $[40,51]$.

(b) OC with thermal noise: Let $\mathbf{G}_{1} \sim \mathcal{C} \mathcal{N}_{R \times T}\left(0_{R \times T}\right.$, $\left.\mathbf{I}_{R}, \mathbf{I}_{T}\right), \mathbf{G}_{2} \sim \mathcal{C} \mathcal{N}_{R \times L}\left(0_{R \times L}, \mathbf{I}_{R}, \mathbf{P}\right)$ with the matrix $\mathbf{P}$ having $L$ positive eigenvalues in descendent order $\left(p_{1}, \ldots, p_{L}\right)$, then the joint PDF of the nonzero eigenvalues of $\mathbf{W}=\mathbf{G}_{1}^{H}\left(\mathbf{G}_{2} \mathbf{G}_{2}^{H}+b \mathbf{I}\right)^{-1} \mathbf{G}_{1}$ is [41]

$$
\begin{array}{r}
f_{\lambda}(\mathbf{x})=K|\boldsymbol{\Phi}(\mathbf{x})||\boldsymbol{\Xi}(\mathbf{x})| \prod_{i=1}^{\min (R, T)} x_{i}^{T-\min (R, T)} e^{-b x_{i}}, \\
\left(\infty>x_{1} \geq \cdots \geq x_{\min (R, T)} \geq 0\right),
\end{array}
$$

where $\lambda=\left(\lambda_{1}, \ldots, \lambda_{\min (R, T)}\right), \mathbf{x}=\left(x_{1}, \ldots\right.$, $\left.x_{\min (R, T)}\right)$, and

$$
K=\frac{(-1)^{R(R-1) / 2}}{\prod_{i=1}^{\min (R, T)}(T-i) ! \prod_{i=1}^{R}(R-i) ! \prod_{1 \leq i<j \leq L}\left(p_{i}-p_{j}\right)},
$$




$$
\begin{aligned}
& \{\boldsymbol{\Phi}(\mathbf{x})\}_{i, j} \\
& \quad\left\{\begin{array}{l}
p_{i}^{j-1}, \\
i=1, \ldots, L ; j=1, \ldots, L-R, \\
p_{i}^{L-j} e^{b / p_{i}} \Gamma\left(R-j+1, \frac{b}{p_{i}}\right), \\
i=1, \ldots, L ; j=L-R+1, \ldots, L-\min (R, T), \\
p_{i}^{L-R-1} e^{b\left(x_{j}+1 / p_{i}\right)} \frac{\Gamma\left(T+1, b x_{j}+b / p_{i}\right)}{\left(x_{j}+1 / p_{i}\right)}, \\
i=1, \ldots, L ; j=L-\min (R, T)+1, \ldots, L,
\end{array}\right. \\
& \left\{\begin{array}{l}
\boldsymbol{\Xi}(\mathbf{x})\}_{i, j}, \quad i, j=1, \ldots, \min (R, T),
\end{array}\right.
\end{aligned}
$$

with $\Gamma(\cdot, \cdot)$ being the upper incomplete Gamma function [50, Equation (8.350.2)].

Again, the joint PDF has the same form as (1). More results can be found in [39].

In summary, the random matrices discussed in Section 1 share a common structure on the joint distributions of their eigenvalues. Based on this common structure, we derive in the following section a general result for the marginal distribution of each ordered eigenvalue.

\section{Marginal Distributions of Ordered Eigenvalues}

\subsection{General Expression for the Marginal Distribution}

Theorem 1. If the joint PDF of the ordered eigenvalues $\left(\lambda_{1}, \ldots, \lambda_{m}\right)$ is given by (1), the marginal CDF of the $k$ th largest eigenvalue $\lambda_{k}$ can be expressed as $(a \leq z \leq b, 1 \leq k \leq m)$

$$
\begin{aligned}
F_{\lambda_{k}}(z)= & K \sum_{l=0}^{k-1}(-1)^{l}\left(\begin{array}{c}
l+m-k \\
l
\end{array}\right) \\
& \times \sum_{\substack{\beta_{1}<\cdots<\beta_{k-l-1} \\
\beta_{k-l}<\cdots<\beta_{m}}}\left|\left\{\begin{array}{cc}
\int_{a}^{b} \phi_{i}(y) \xi_{j}(y) \nu(y) d y, & i=1, \ldots, n ; j=\beta_{1}, \ldots, \beta_{k-l-1} . \\
\int_{a}^{z} \phi_{i}(y) \xi_{j}(y) \nu(y) d y, & i=1, \ldots, n ; j=\beta_{k-l}, \ldots, \beta_{m} . \\
\phi_{i, j}, & i=1, \ldots, n ; j=m+1, \ldots, n .
\end{array}\right\}\right|,
\end{aligned}
$$

where $\left(\begin{array}{c}n \\ m\end{array}\right)=n ! / m ! /(n-m) !, \boldsymbol{\beta}=\left(\beta_{1}, \ldots, \beta_{m}\right)$ is a permutation of $(1, \ldots, m)$ that satisfies $\beta_{1}<\cdots<\beta_{k-l-1}$ and $\beta_{k-l}<\cdots<\beta_{m}$. The second summation is over all permutations, that is, $\left(\begin{array}{c}m \\ k-l-1\end{array}\right)$ in total.

\section{Proof. See Appendix B.}

Given the marginal CDF, the corresponding marginal PDF is easy to obtain, given the well-known result on the derivative of a determinant [52, Equation (6.1.19)],

$$
\frac{\mathrm{d}|\mathbf{A}(x)|}{\mathrm{d} x}=\sum_{q=1}^{n}\left|\mathbf{A}_{q}(x)\right|,
$$

where $\mathbf{A}(x)$ is an $n \times n$ matrix with each element being a function of $x$, and $\mathbf{A}_{q}(x)$ is identical to $\mathbf{A}(x)$, except that all elements in the $q$ th column are replaced by their derivatives with respect to $x$.

In the literature, exact expressions on the marginal distributions of the ordered eigenvalues were reported in $[20,45,46]$. (The expression obtained in $[45,46]$ was given in the form of a sum of $x^{\alpha} e^{-\beta x}$ terms. That form allows closed-form evaluation of moments and characteristic functions of the eigenvalues.) These results, however, needed separate expressions to represent the eigenvalue distributions of Wishart (i.e., $n=m$ ) and pseudo-Wishart (i.e., $n>m$ ) matrices. In contrast, Theorem 1 unifies the two cases $(n=m$ and $n>m$ ) with only a single expression. It is also worth noting that, although another unified expression could be found in [21], the result there was given in terms of the determinant of rank-3 tensor $M$. (Letting $\mathbf{A}$ be a rank-3 tensor, that is, $\{\mathbf{A}\}_{i, j, k}=a_{i, j, k}$ for $i, j, k=1, \ldots, N$, the "determinant" of $A$, denoted by $\mathcal{T}(\mathbf{A})$, is given by [7] $T(\mathbf{A}) \triangleq \sum_{\boldsymbol{\alpha}} \operatorname{sgn}(\boldsymbol{\alpha}) \sum_{\boldsymbol{\beta}} \operatorname{sgn}(\boldsymbol{\beta}) \prod_{k=1}^{N} a_{\alpha_{k}, \beta_{k}, k}$, where $\boldsymbol{\alpha}$ and $\boldsymbol{\beta}$ are permutations of the integers $(1, \ldots, N)$, the summation is over all possible permutations, and $\operatorname{sgn}(\cdot)$ is the sign of the permutation.) which was computationally complex, especially comparing to our new result in a conventional (2dimensional) determinant form. Perhaps the most related work in the literature is [41]. To see the difference between [41] and Theorem 1 above, we rewrite [41, Lemma 1] in the following proposition. After comparing the two results, one can clearly see that our expression is much more efficient in computational complexity, since the functions $\int_{z}^{b} \mathrm{~d} y$ in (15) are replaced by constant numbers $\int_{a}^{b} \mathrm{~d} y$ in (13). 
Proposition 2. The marginal CDF of $\lambda_{k}$ can be alteratively expressed as

$$
F_{\lambda_{k}}(z)=K \sum_{\substack{l=0 \\
\beta_{1}<\cdots<\beta_{k-l-1} \\
\beta_{k-l}<\cdots<\beta_{m}}}^{k-1}\left|\left\{\begin{array}{cc}
\int_{z}^{b} \phi_{i}(y) \xi_{j}(y) \nu(y) \mathrm{d} y, & i=1, \ldots, n ; j=\beta_{1}, \ldots, \beta_{k-l-1} . \\
\int_{a}^{z} \phi_{i}(y) \xi_{j}(y) \nu(y) \mathrm{d} y, & i=1, \ldots, n ; j=\beta_{k-l}, \ldots, \beta_{m} . \\
\phi_{i, j}, & i=1, \ldots, n ; j=m+1, \ldots, n .
\end{array}\right\}\right| .
$$

Proof. By the definition of marginal CDF, we have

$$
\begin{aligned}
F_{\lambda_{k}}(z) & =\operatorname{Pr}\left(z \geq \lambda_{k}\right) \\
& =\sum_{l=0}^{k-1} \operatorname{Pr}\left(\lambda_{1} \geq \cdots \geq \lambda_{k-l-1} \geq z \geq \lambda_{k-l} \geq \cdots \geq \lambda_{m}\right) \\
& =\sum_{l=0}^{k-1} \int_{D_{l}} f_{\lambda}(\mathbf{x}) \mathrm{d} \mathbf{x},
\end{aligned}
$$

where $D_{l}=\left\{b \geq x_{1} \geq \cdots \geq x_{k-l-1} \geq z \geq x_{k-l} \geq\right.$ $\left.\cdots \geq x_{M} \geq a\right\}$. Substituting (1) into (17) and invoking the generalized Cauchy-Binet formula [41, Lemma 1] the multinested integration can be carried out analytically. As such, we get the desired result.

It is also worth noting that the work of this paper can be viewed as an interesting proof for the equivalence between (13) and (15), because both Theorem 1 and Proposition 2 represent the same eigenvalue distribution.

3.2. Specific Eigenvalue Distributions. As a simple application of the general result, we particularize into the eigenvalue distribution of the double-scattering channel matrix.

Corollary 1. Given that the ordered eigenvalues $\lambda$ of $\mathbf{W}$ (= $\mathbf{G}_{2}^{H} \mathbf{G}_{1}^{H} \mathbf{G}_{1} \mathbf{G}_{2} / N_{s}$ ) are jointly distributed as (5), the marginal CDF of the kth largest eigenvalue $\lambda_{k}$ can be expressed as

$$
\begin{aligned}
& F_{\lambda_{k}}(z)=K \sum_{l=0}^{k-1}(-1)^{l}\left(\begin{array}{c}
l+M-k \\
l
\end{array}\right) \\
& \times \sum_{\substack{\beta_{1}<\cdots<\beta_{k-l-1} \\
\beta_{k-l}<\cdots<\beta_{M}}}|\Upsilon(z, l, \beta)|, \quad(1 \leq k \leq M ; z \geq 0),
\end{aligned}
$$

where $K$ is given in (7), the second summation is over all combinations of $\left(\beta_{1}<\cdots<\beta_{k-l-1}\right)$ and $\left(\beta_{k-l}<\cdots<\beta_{M}\right)$ with $\beta=\left(\beta_{1}, \ldots, \beta_{M}\right)$ being a permutation of $(1, \ldots, M)$, and

$$
\begin{aligned}
& \{\mathbf{\Upsilon}(z, l, \boldsymbol{\beta})\}_{i, j} \\
& =\left\{\begin{array}{c}
h\left(\infty, T-N_{t}+i-2, \frac{1}{N_{s}}, N-S+j-1\right), \\
i=1, \ldots, S ; j=\beta_{1}, \ldots, \beta_{k-l-1} . \\
h\left(z, T-N_{t}+i-2, \frac{1}{N_{s}}, N-S+j-1\right) \\
i=1, \ldots, S ; j=\beta_{k-l}, \ldots, \beta_{M} . \\
(-1)^{S-j}(T-M-N+i+j-2) ! N_{s}^{-(T-M-N+i+j-1)}, \\
i=1, \ldots, S ; j=M+1, \ldots, S .
\end{array}\right.
\end{aligned}
$$

with

$$
\begin{aligned}
& h(z, a, b, c) \\
& =c !\left[(a+c+1) ! b^{a+c+2}\right. \\
& \left.\quad-\sum_{n=0}^{c} \frac{2 b^{(a+c+2-n) / 2}}{n !} z^{(a+c+2+n) / 2} K_{a+c+2-n}\left(2 \sqrt{\frac{z}{b}}\right)\right] \\
& \quad h(\infty, a, b, c)=c !(a+c+1) ! b^{a+c+2} .
\end{aligned}
$$

Proof. Define

$$
\begin{array}{r}
h(z, a, b, c)=2 b^{(a+1) / 2} \int_{0}^{z} x^{c+(a+1) / 2} K_{a+1}\left(2 \sqrt{\frac{x}{b}}\right) \mathrm{d} x, \\
(a \in \mathbb{Z}, \mathbb{}, 1 \in \mathbb{N}) .
\end{array}
$$

The integral above can be written in a closed form by invoking [50, Equations (8.352.1) and (8.432.6)]. The results are given in (20). Substituting (5) into (13) and using (21) completes the proof.

The marginal CDF of the largest eigenvalue (i.e., $\lambda_{1}$ ) of the double-scattering channel matrix was reported earlier in [14]. The expression above extends this result to marginal distributions of all ordered eigenvalues. We also note that marginal CDFs of the ordered eigenvalues were also investigated in the authors' previous work [47]. However, the result there were derived based on Proposition 2 above. 


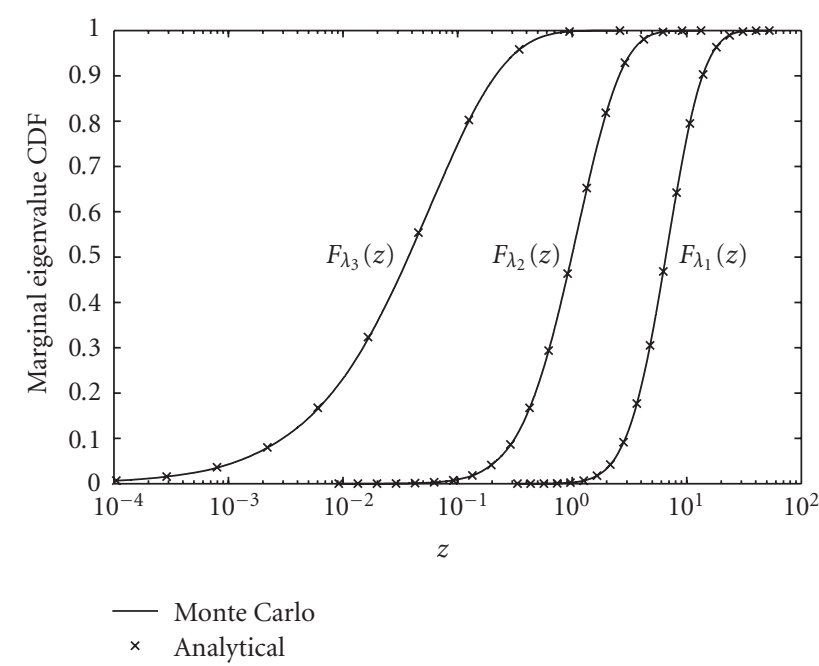

FIgure 1: Marginal CDFs of ordered eigenvalues of $\mathbf{W}=$ $\mathbf{G}_{1}^{H} \mathbf{G}_{2}^{H} \mathbf{G}_{2} \mathbf{G}_{1} / N_{s}$ when $N_{r}=3, N_{s}=3$, and $N_{t}=3$.

In Figure 1, we plot the eigenvalue CDFs of the matrix $\mathbf{W}\left(=\mathbf{G}_{1}^{H} \mathbf{G}_{2}^{H} \mathbf{G}_{2} \mathbf{G}_{1} / N_{s}\right)$, when $N_{r}=3, N_{s}=3$, and $N_{t}=3$. The analytical results are computed by (18), and the Monte Carlo results are based on $10^{6}$ channel realizations. A perfect agreement is observed between the analytical and Monte Carlo curves.

\section{Performance Analysis of MIMO SVD Systems}

In this section, we consider performance analysis of MIMO SVD systems. Uncorrelated double-scattering fading channels are assumed, where the MIMO channel matrix $\mathbf{H}$ is modeled as [14] (the double-scattering channel considered here was also termed the Rayleigh-product channel [14])

$$
\mathbf{H}=\frac{1}{\sqrt{N_{s}}} \mathbf{G}_{1} \mathbf{G}_{2}
$$

where $\mathbf{G}_{1} \sim \quad \mathcal{C} \mathcal{N}_{N_{r} \times N_{s}}\left(0_{N_{r} \times N_{s}}, \mathbf{I}_{N_{r}}, \mathbf{I}_{N_{s}}\right), \quad \mathbf{G}_{2} \sim$ $\mathcal{C} \mathcal{N}_{N_{s} \times N_{t}}\left(0_{N_{s} \times N_{t}}, \mathbf{I}_{N_{s}}, \mathbf{I}_{N_{t}}\right), N_{t}, N_{r}$, and $N_{s}$ are the numbers of transmit antennas, receive antennas, and the scatterers, respectively. The matrix $\mathbf{G}_{2}$ represents the fading channel between the transmitter and the scatterers, while $\mathbf{G}_{1}$ represents the channel between the scatterers and the receiver. The introduction of the double-scattering model is due to the fact that [53] MIMO channels exhibits a rank deficient behavior when there is not enough scattering around the transmitter and receiver (a typical example is the keyhole/pinhole channel [54], where the MIMO channel matrix has rank one regardless of the number of transmit and receive antennas, since only one scatterer exists in the environment). In this model, the MIMO channel matrix is characterized by the product (concatenation) of two Gaussian matrices, representing the channel from the transmitter to the scatterers, and the channel from the scatterers to the receiver, respectively. Varying the number of the scatterers, the double-scattering model describes a broad family of practical channels, ranging from conventional
Rayleigh channel (infinite scatterers) to degenerate keyhole channel (only one scatterer). In the rest of this section, we use notations $S, T, M$, and $N$ as they were defined in (6).

4.1. System Model. Consider a MIMO channel with $N_{t}$ transmit and $N_{\mathbf{r}}$ receive antennas. The received vector $\mathbf{r}$ can be expressed as

$$
\mathbf{r}=\mathbf{H s}+\mathbf{n},
$$

where $\mathbf{H} \in \mathbb{C}^{N_{r} \times N_{t}}$ is the channel matrix, $\mathbf{s} \in \mathbb{C}^{N_{t} \times 1}$ is the vector of signals transmitted, and $\mathbf{n} \in \mathbb{C}^{N_{t} \times 1}$ is the complex additive white Gaussian noise (AWGN) vector with zero mean and identity covariance matrix. In MIMO SVD, assuming perfect channel state information (CSI) at the transmitter, the transmit vector $\mathbf{s}$ is formed by mapping $L$ ( $\leq$ $M)$ modulated symbols $\mathbf{d}\left(\triangleq\left(d_{1}, \ldots, d_{L}\right)^{T}\right)$ onto $N_{t}$ transmit antennas via a linear precoding:

$$
\mathbf{s}=\mathbf{P d}
$$

where $\mathbf{P} \in \mathbb{C}^{N_{t} \times L}$ is the spatial pre-coding matrix. Here, the columns of $\mathbf{P}$ are the right singular vectors of $\mathbf{H}$ corresponding to the $L$ largest singular values. Under the assumption of perfect CSI at the receiver, the decision statistics of MIMO SVD, denoted by $\hat{\mathbf{d}}\left(\triangleq\left(\hat{d}_{1}, \ldots, \hat{d}_{L}\right)^{T}\right)$, is obtained by weighting the receive signal $\mathbf{r}$ with a spatial equalizing matrix $\mathbf{Q} \in \mathbb{C}^{N_{r} \times L}$

$$
\widehat{\mathbf{d}}=\mathbf{Q}^{H} \mathbf{r}
$$

where the columns of $\mathbf{Q}$ are the left singular vectors of $\mathbf{H}$ corresponding to the $L$ largest singular values. After such precoding and equalization, the MIMO channel is decomposed into a set of equivalent single-input single-output (SISO) channels, whose input-output relation is $(k=1, \ldots, L)$

$$
\hat{d}_{k}=\sqrt{\lambda_{k}} d_{k}+n_{k}
$$

where $\lambda_{k}$ is the $k$ th largest eigenvalue of $\mathbf{H}^{H} \mathbf{H}$, and $n_{k}$ is the complex AWGN with zero mean and unit variance (i.e., 0.5 variance per complex dimension). Hereafter, we term these SISO channels as the sub-channels of MIMO SVD. Letting $\rho_{k}$ denote the power allocated to the $k$ th subchannel, the instantaneous SNR of the $k$ th subchannel can be expressed as $(k=1, \ldots, L)$

$$
\gamma_{k}=\rho_{k} \lambda_{k}
$$

Clearly, the performance of MIMO SVD depends directly on the eigenvalues $\lambda_{k} s$.

It is worth noting that, although the capacity-achievable power allocation for MIMO SVD is water-filling [6], exact analysis of such allocation strategy is very difficult (in water-filling, each allocated power $\rho_{k}$ is a function of all eigenvalues $\lambda$, leading to an intractable SER expression of each subchannel [18], Ft. 1). For this reason, earlier researches on MIMO SVD generally considered fixed (but not necessarily uniform) power allocation $[18,20]$. (Indeed, 
given a sufficiently high SNR, the water-filling power strategy tends to a uniform power allocation, that is, a special case of the fixed allocation [20].) Following this direction, we consider here fixed power allocation, but it worth noting hat the results obtained can serve as a starting point for the analysis of channel-dependent power allocations [19], as well as the analysis of diversity-multiplexing tradeoff [55].

4.2. Performance Analysis. First of all, we consider the outage performance of MIMO SVD. The outage probability, as an important measure of service quality, is defined by the probability that the received SNR drops below an acceptable threshold $\gamma_{\text {th }}$. For convenience sake, we assume equal power allocation is employed, that is, $\rho_{1}=\cdots=\rho_{L}=\rho / L$ with $\rho$ denoting the total transmit power (normalized by the noise variance). As such, the SNRs of the subchannels are ordered as $\gamma_{1}>\cdots>\gamma_{L}$, and the outage probability of the overall system is dominated by the worst subchannel (corresponding to $\lambda_{L}$ ). The exact expression on outage probability is then obtained by substituting the CDF (18) into the equation below

$$
\begin{aligned}
P_{\text {out }}(\rho) & =\operatorname{Pr}\left(\gamma_{L}<\gamma_{\text {th }}\right) \\
& =F_{\lambda_{L}}\left(\frac{\gamma_{\text {th }} L}{\rho}\right) .
\end{aligned}
$$

Next, we consider the SER of MIMO SVD. Given the average SER of many general modulation formats (BPSK, BFSK, $M$-PAM, etc.) [56] ((30) also provides good approximations to the SERs of other modulation formats, such as $M$-PSK [56, Equation (5-2-61)])

$$
\mathrm{SER}=\mathbb{E}_{\gamma}[\alpha Q(\sqrt{2 \beta \gamma})],
$$

where $\gamma$ is the instantaneous $\mathrm{SNR}, Q(\cdot)$ is the Gaussian $Q-$ function, $\alpha$ and $\beta$ are modulation-specific constants (e.g., $\alpha=1, \beta=1$ for BPSK), the average SER of the $k$ th subchannel of the MIMO SVD system can be expressed as (after some algebraic manipulations)

$$
\mathrm{SER}_{k}=\frac{\alpha \sqrt{\beta}}{2 \sqrt{\pi}} \int_{0}^{\infty} x^{-1 / 2} e^{-\beta x} F_{\lambda_{k}}\left(\frac{x}{\rho_{k}}\right) \mathrm{d} x, \quad(k=1, \ldots, L) .
$$

Substituting (18) into (31) yields the analytical expression for the average SER. Although deriving a closed-form result for (31) seems difficult, the expression above can be evaluated numerically, which is more efficient than running Monte Carlo simulations. Since independent signals are sent over different subchannels, the global SER (i.e., the average SER of the overall system) can be obtained by averaging the SERs of the active subchannels $[18,19]$

$$
\mathrm{SER}_{\text {global }}=\sum_{k=1}^{L} \frac{\mathrm{SER}_{k}}{L} .
$$

4.3. Numerical Examples. In this subsection, numerical simulations are used to verify the theoretical results above.

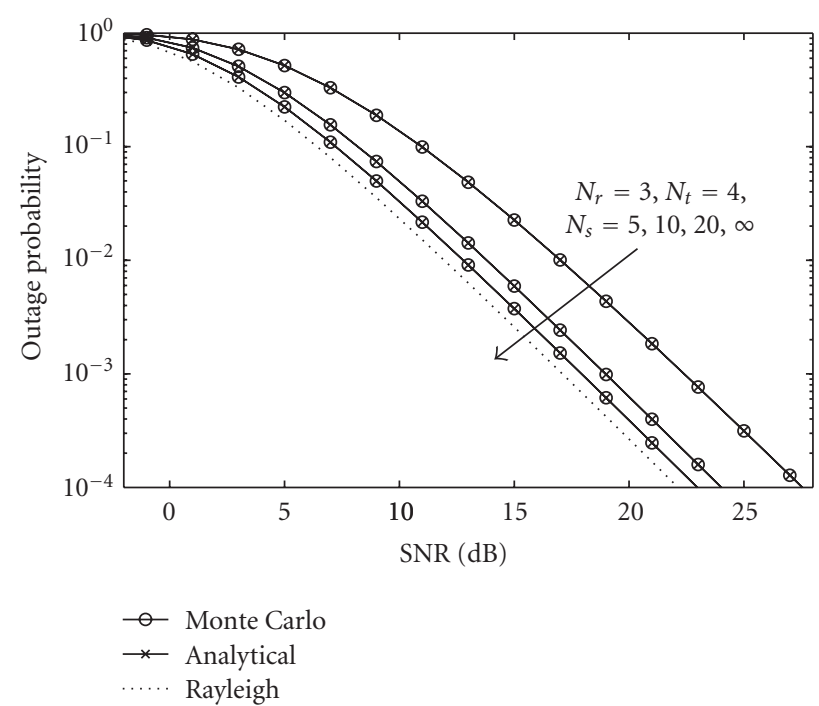

FIGURE 2: Comparisons on outage probabilities of MIMO SVD in different channels: $(3,5,4),(3,10,4),(3,20,4)$, and $(3, \infty, 4)$.

For notational convenience, we denote the double-scattering channel with $N_{t}$ transmit antennas, $N_{r}$ receive antennas, and $N_{s}$ scatterers by a three-tuple $\left(N_{r}, N_{s}, N_{t}\right)$. We also assume that all subchannels are active (i.e., $L=M$ ), upon which equal power allocation is employed (i.e., $\rho_{k}=\rho / M$ for all $k)$.

In Figure 2, we fix the SNR threshold at $\gamma_{\text {th }}=-5 \mathrm{~dB}$ to evaluate the impact of scatterer insufficiency on the outage probability of MIMO SVD. Three channel configurations are considered: $(3,5,4),(3,10,4)$, and $(3,20,4)$. Results from standard Rayleigh channel (i.e., $(3, \infty, 4))$ is also provided for the purpose of comparison. The analytical results are computed with (29), and each Monte Carlo result is based on $10^{6}$ channel realizations. From the figure, we observe an exact agreement between the analytical and Monte Carlo curves. Also, we observe that the lack of scattering certainly degrade the performance of the system, which is consistent with our intuition.

In Figure 3, we plot the SERs of the MIMO SVD subchannels in a $(4,4,3)$ double-scattering channel, using uncoded BPSK modulation. It is shown that all analytical results agree with the Monte-Carlo curves perfectly. It is also observed that the first and second strongest subchannels outperform the third subchannel significantly. This indicates that further improvements (in SER) is possible if only a subset of subchannels is used. In-depth analysis along this direction can be found in [57] on the linear transceiver design with adaptive number of sub-streams, and also in [55] on the fundamental tradeoff between diversity and multiplexing of MIMO SVD (note that both papers assumed conventional Rayleigh/Rican fading).

\section{Conclusion}

The eigenvalue distribution of random matrices has long been known as a powerful tool for analyzing and designing 


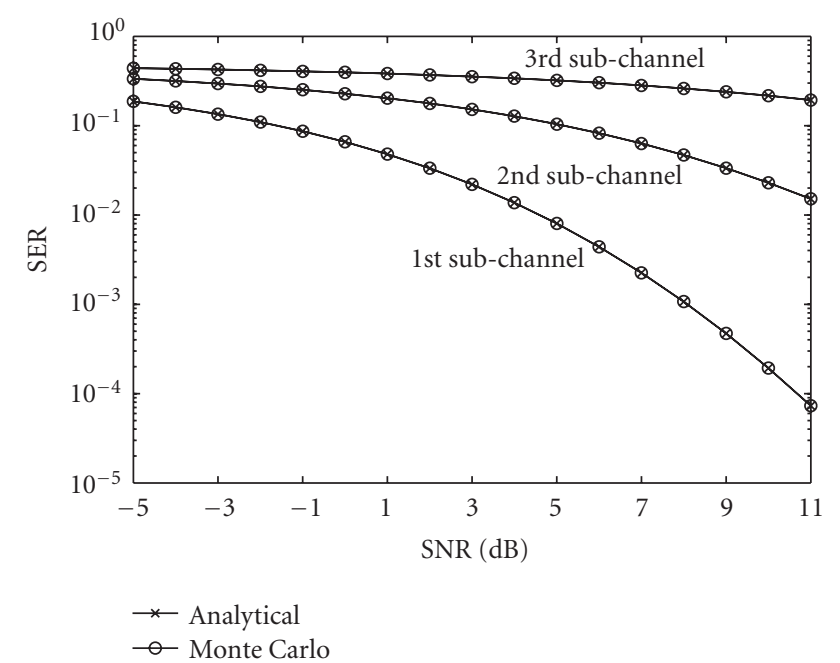

FIGURE 3: Subchannel SER of MIMO SVD in a $(4,4,3)$ doublescattering channel when uncoded BPSK is used.

communication systems. In this paper, we derived a new expression for the marginal distributions of the ordered eigenvalues of certain important random matrices. The new expression was compacter in representation and more efficient in computational complexity, when comparing to existing results in the literature. As an illustrative application, we then used the general result to analyze the performance of MIMO SVD systems, under the assumption of doublescattering fading channels. Joint and marginal eigenvalue distributions of the channel matrix were presented, which further yielded analytical expressions on the average SER and outage probability of the system. Finally, the theoretical results were verified with numerical simulations.

\section{Appendices}

\section{A. Proof for the Joint Eigenvalue Distribution}

Recall that $\mathbf{W}=\mathbf{G}_{2}^{H} \mathbf{G}_{1}^{H} \mathbf{G}_{1} \mathbf{G}_{2} / N_{s}, \quad \boldsymbol{\lambda}=\left(\lambda_{1}, \ldots, \lambda_{M}\right)$ are the nonzero descendingly ordered eigenvalues of $\mathbf{W}$, with $S, T$, $M$, and $N$ being given by (6). We also define the following new notations $\mathbf{Y}=\mathbf{G}_{1}^{H} \mathbf{G}_{1} / N_{s}$ with $\boldsymbol{\eta}=\left(\eta_{1}, \ldots, \eta_{S}\right)$ being its nonzero descendingly ordered eigenvalues. Then, we take three steps to get the joint PDF of $\lambda$. First of all, we get the joint PDF of $\boldsymbol{\eta}$, that is, $f_{\boldsymbol{\eta}}(\mathbf{y})$. Next, we obtain the joint PDF of $\lambda$ conditioned on $\boldsymbol{\eta}$, that is, $f_{\lambda \mid \eta}(\mathbf{x} \mid \mathbf{y})$. Finally, we average the conditional joint PDF $f_{\lambda \mid \eta}(\mathbf{x} \mid \mathbf{y})$ over $\boldsymbol{\eta}$ to get the unconditional joint $\operatorname{PDF} f_{\lambda}(\mathbf{x})$. Details on this conditionand-average procedure are given below.

(i) Get the joint PDF of the nonzero ordered eigenvalues $\boldsymbol{\eta}$ of $\mathbf{Y}\left(=\mathbf{G}_{1}^{H} \mathbf{G}_{1} / N_{s}\right)$. Based on the result of [6], we have

$$
\begin{array}{r}
f_{\eta}(\mathbf{y})=K_{1} N_{s}^{S T}|\mathbf{V}(\mathbf{y})|^{2} \prod_{i=1}^{S} y_{i}^{T-S} e^{-N_{s} y_{i}}, \\
\left(y_{1} \geq y_{2} \geq \cdots \geq y_{S} \geq 0\right),
\end{array}
$$

where

$$
\begin{gathered}
K_{1}=\frac{1}{\prod_{i=1}^{S}(S-i) !(T-i) !}, \\
\{\mathbf{V}(\mathbf{y})\}_{i, j}=y_{i}^{j-1}, \quad i, j=1, \ldots, S .
\end{gathered}
$$

(ii) Get the joint PDF of $\lambda$, conditioned on $\boldsymbol{\eta}$. To this end, we note that if $\mathbf{Y}$ is rank deficient, (i.e., $N_{r}<$ $\left.N_{s}\right), \lambda$ are the eigenvalues of $\widetilde{\mathbf{G}}_{2}^{H} \mathbf{D}_{\mathbf{Y}} \widetilde{\mathbf{G}}_{2}$, where $\mathbf{D}_{\mathbf{Y}}$ is a diagonal matrix with $\boldsymbol{\eta}$ as its diagonal elements, and $\widetilde{\mathbf{G}}_{2} \in \mathbb{C}^{N_{\mathrm{t}} \times S}$ is a complex Gaussian matrix with statistically independent, zero-mean, unit-variance elements. Knowing this, we get the conditional joint PDF of $\lambda$ by invoking [47, Lemma 2]:

$$
\begin{array}{r}
f_{\lambda \mid \eta}(\mathbf{x} \mid \mathbf{y})=\frac{K_{2}}{|\mathbf{U}(\mathbf{y})| \prod_{i=1}^{S} y_{i}^{N_{t}}}|\mathbf{E}(\mathbf{x}, \mathbf{y})||\mathbf{\Xi}(\mathbf{x})| \prod_{i=1}^{M} x_{i}^{N-S}, \\
\left(x_{1} \geq x_{2} \geq \cdots \geq x_{M} \geq 0\right),
\end{array}
$$

where

$$
\begin{gathered}
K_{2}=\frac{(-1)^{(S-M)(S+M-1) / 2}}{\prod_{i=1}^{M}\left(N_{t}-i\right) !}, \\
\{\mathbf{U}(\mathbf{y})\}_{i, j}=\left(-\frac{1}{y_{i}}\right)^{j-1}, \quad i, j=1, \ldots, S . \\
\{\mathbf{E}(\mathbf{x}, \mathbf{y})\}_{i, j}=\left\{\begin{array}{lc}
e^{-x_{j} / y_{i}}, & i=1, \ldots, S ; j=1, \ldots, M . \\
\left(-\frac{1}{y_{i}}\right)^{S-j}, & i=1, \ldots, S ; j=M+1, \ldots, S . \\
\{\mathbf{\Xi}(\mathbf{x})\}_{i, j}=x_{i}^{j-1}, & i, j=1, \ldots, M .
\end{array}\right.
\end{gathered}
$$

By invoking the identity [14, Equation (74)]

$$
|\mathbf{U}(\mathbf{y})|=|\mathbf{V}(\mathbf{y})| \prod_{i=1}^{S} y_{i}^{1-S}
$$

we rewrite (A.3) as follows:

$$
f_{\lambda \mid \eta}(\mathbf{x} \mid \mathbf{y})=\frac{K_{2}}{|\mathbf{V}(\mathbf{y})| \prod_{i=1}^{S} y_{i}^{1+N_{t}-S}}|\mathbf{E}(\mathbf{x}, \mathbf{y})||\mathbf{\Xi}(\mathbf{x})| \prod_{i=1}^{M} x_{i}^{N-S} .
$$

(iii) Get the unconditional joint PDF of $\lambda$ by averaging conditional PDF over $\boldsymbol{\eta}$

$$
\begin{aligned}
f_{\lambda}(\mathbf{x})= & \int_{D} f_{\lambda \mid \eta}(\mathbf{x} \mid \mathbf{y}) f_{\eta}(\mathbf{y}) \mathrm{d} \mathbf{y} \\
= & K_{1} K_{2} N_{s}^{S T} \\
& \quad \times \int_{D}|\mathbf{E}(\mathbf{x}, \mathbf{y})||\mathbf{V}(\mathbf{y})| \\
& \quad \times \prod_{i=1}^{S} y_{i}^{T-N_{t}-1} e^{-N_{s} y_{i}} \mathrm{~d} \mathbf{y}|\mathbf{\Xi}(\mathbf{x})| \prod_{i=1}^{M} x_{i}^{N-S},
\end{aligned}
$$


where $D=\left\{\left(y_{1}, y_{2}, \ldots, y_{S}\right): y_{1} \geq y_{2} \geq \cdots \geq y_{S}>\right.$ $0\}$, and $\mathrm{dy}=\mathrm{d} y_{1} \mathrm{~d} y_{2} \cdots \mathrm{d} y_{S}$. The integration above can be evaluated in a closed form with the generalized Cauchy-Binet formula (see, e.g., [7, Corollary 2]). We finally arrive at the expression below

$$
f_{\lambda}(\mathbf{x})=K_{1} K_{2} N_{s}^{S T}|\boldsymbol{\Phi}(\mathbf{x})||\boldsymbol{\Xi}(\mathbf{x})| \prod_{i=1}^{M} x_{i}^{N-S},
$$

with

$$
\{\boldsymbol{\Phi}(\mathbf{x})\}_{i, j}=\left\{\begin{array}{l}
\int_{0}^{\infty} y^{T-N_{t}+i-2} e^{-x_{j} / y-N_{s} y} \mathrm{~d} y, \\
i=1, \ldots, S ; j=1, \ldots, M . \\
\int_{0}^{\infty}(-1)^{S-j} y^{T-M-N+i+j-2} e^{-N_{s} y} \mathrm{~d} y, \\
i=1, \ldots, S ; j=M+1, \ldots, S .
\end{array}\right.
$$

The proof is completed by the use of [50, Equation $(8.432 .6)]$

$$
\begin{array}{r}
\int_{0}^{\infty} x^{a} e^{-x / b-c / x} \mathrm{~d} x=2(b c)^{(a+1) / 2} K_{a+1}\left(2 \sqrt{\frac{c}{b}}\right), \\
a \in \mathbb{R}, b>0, c>0 .
\end{array}
$$

\section{B. Proof of Theorem 1}

Let $\bar{\lambda}=\left(\bar{\lambda}_{1}, \bar{\lambda}_{2}, \ldots, \bar{\lambda}_{m}\right)$ denote the unordered version of $\lambda$. Then, by the symmetry of (1), we get the joint PDF of $\bar{\lambda}$

$$
\begin{array}{r}
f_{\bar{\lambda}}(\mathbf{x})=\frac{K}{m !}|\boldsymbol{\Phi}(\mathbf{x})||\boldsymbol{\Xi}(\mathbf{x})| \prod_{i=1}^{m} \nu\left(x_{i}\right), \\
\left(b \geq x_{j} \geq a, \quad j=1, \ldots, m\right) .
\end{array}
$$

Note that the coefficient $1 / m$ ! is due to the change in function domains when comparing with (1). This joint PDF can be simplified as follows:

$$
f_{\bar{\lambda}}(\mathbf{x})=\frac{K}{m !}|\boldsymbol{\Phi}(\mathbf{x})||\Psi(\mathbf{x})|,
$$

where $\Psi(\mathbf{x})$ is an $n \times n$ matrix defined by

$$
\{\boldsymbol{\Psi}(\mathbf{x})\}_{i, j}= \begin{cases}\psi_{j}\left(x_{i}\right), & i, j=1, \ldots, m . \\ 1, & i=j=m+1, \ldots, N . \\ 0, & \text { otherwise. }\end{cases}
$$

With $\psi_{j}\left(x_{i}\right)=\xi_{j}\left(x_{i}\right) \nu\left(x_{i}\right)$. The usefulness of this form will become apparent immediately.

Next, we rewrite the joint PDF of $\bar{\lambda}$ by using the fact that $|\mathbf{A}||\mathbf{B}|=|\mathbf{A B}|$, with $\mathbf{A}$ and $\mathbf{B}$ being two square matrices of the same size (a similar method was used in $[58,59]$ to derive the distributions of eigenvalue subsets of Wishart matrices):

$$
\begin{aligned}
& f_{\bar{\lambda}}(\mathbf{x}) \\
& =\frac{K}{m !}\left|\left\{\begin{array}{cc}
\sum_{\alpha=1}^{m} \phi_{i}\left(x_{\alpha}\right) \psi_{j}\left(x_{\alpha}\right), & i=1, \ldots, n ; j=1, \ldots, m . \\
\phi_{i, j}, & i=1, \ldots, n ; j=m+1, \ldots, n .
\end{array}\right\}\right|
\end{aligned}
$$

Using the multilinear property of the determinant, we further simplify the joint PDF as

$$
\begin{aligned}
& f_{\bar{\lambda}}(\mathbf{x}) \\
& =\frac{K}{m !} \sum_{\boldsymbol{\alpha}}\left|\left\{\begin{array}{cc}
\phi_{i}\left(x_{\alpha_{j}}\right) \psi_{j}\left(x_{\alpha_{j}}\right), & i=1, \ldots, n ; j=1, \ldots, m . \\
\phi_{i, j}, & i=1, \ldots, n ; j=m+1, \ldots, n .
\end{array}\right\}\right|,
\end{aligned}
$$

where $\boldsymbol{\alpha}=\left(\alpha_{1}, \ldots, \alpha_{m}\right)$ is a permutation of $(1, \ldots, m)$, and the summation is over all permutations. The usefulness of the joint PDF in this form will become apparent immediately.

According to [60, Equation (3.4.3)], the marginal CDF of the $k$ th largest variable $\lambda_{k}$ can be expressed as (note that $[60$, Equation (3.4.3)] deals with random variables in ascendent order. However, the result there can be easily rewritten to cover the descending-order cases by appropriate change of variables)

$$
F_{\lambda_{k}}(z)=\sum_{l=0}^{k-1}(-1)^{l}\left(\begin{array}{c}
l+m-k \\
l
\end{array}\right)\left(\begin{array}{c}
m \\
l+m+1-k
\end{array}\right) F_{\zeta_{l, k}}(z)
$$

with

$$
\zeta_{l, k} \triangleq \max \left(\bar{\lambda}_{1}, \bar{\lambda}_{2}, \ldots, \bar{\lambda}_{l+m+1-k}\right)
$$

and $F_{\zeta_{l, k}}(\cdot)$ being the CDF of $\zeta_{l, k}$. Obviously, the desired marginal CDF $F_{\lambda_{k}}(z)$ depends directly on an intermediate CDF $F_{\zeta, k}(\cdot)$. As we show below, this intermediate CDF can be obtained by the use of the joint PDF in (B.5)

$$
\begin{aligned}
F_{\zeta_{l, k}}(z)= & \operatorname{Pr}\left\{\max \left(\bar{\lambda}_{1}, \ldots, \bar{\lambda}_{l+m+1-k}\right) \leq z\right\} \\
= & \int_{a}^{z} \mathrm{~d} x_{1} \cdots \int_{a}^{z} \mathrm{~d} x_{l+m+1-k} \\
& \times \int_{a}^{b} \mathrm{~d} x_{l+m+2-k} \cdots \int_{a}^{b} f_{\bar{\lambda}}(\mathbf{x}) \mathrm{d} x_{m} .
\end{aligned}
$$

Substituting (B.5) into (B.9) and simplifying yields

$$
\begin{aligned}
& F_{\zeta, k}(z) \\
& =\frac{K}{m !} \sum_{\alpha}\left|\left\{\begin{array}{c}
\int_{a}^{z} \phi_{i}\left(x_{\alpha_{j}}\right) \psi_{j}\left(x_{\alpha_{j}}\right) \mathrm{d} x_{\alpha_{j}}, \\
i=1, \ldots, n ; j=\beta_{1}, \ldots, \beta_{l+m+1-k .} \\
\int_{a}^{b} \phi_{i}\left(x_{\alpha_{j}}\right) \psi_{j}\left(x_{\alpha_{j}}\right) \mathrm{d} x_{\alpha_{j}}, \\
i=1, \ldots, n ; j=\beta_{l+m+2-k}, \ldots, \beta_{m} . \\
\phi_{i, j}, \\
i=1, \ldots, n ; j=m+1, \ldots, n .
\end{array}\right\}\right|,
\end{aligned}
$$

where $\alpha_{\beta_{t}}=t$ for $t=1, \ldots, m$, that is, $\left(\beta_{1}, \ldots, \beta_{m}\right)$ are the indices of $(1, \ldots, m)$ in the permutations. Noticing that all integrals above are independent of the order of $\alpha_{j}(j=$ $\beta_{1}, \ldots, \beta_{l+m+1-k}$ and $j=\beta_{l+m+2-k}, \ldots, \beta_{m}$, resp.), we can further simplify the summation as 


$$
\begin{aligned}
F_{\zeta, k}(z)= & K \frac{(l+m+1-k) !(k-l-1) !}{m !} \\
& \times \sum_{\substack{\beta_{1}<\cdots<\beta_{l+m+1-k} \\
\beta_{l+m+2-k}<\cdots<\beta_{m}}} \mid\left\{\begin{array}{cc}
\int_{a}^{z} \phi_{i}(y) \psi_{j}(y) \mathrm{d} y, & i=1, \ldots, n ; j=\beta_{1}, \ldots, \beta_{l+m+1-k \cdot} \\
\int_{a}^{b} \phi_{i}(y) \psi_{j}(y) \mathrm{d} y, & i=1, \ldots, n ; j=\beta_{l+m+2-k}, \ldots, \beta_{m} \cdot \\
\phi_{i, j}, & i=1, \ldots, n ; j=m+1, \ldots, n .
\end{array}\right\} .
\end{aligned}
$$

Here, we abuse the notation $\beta=\left(\beta_{1}, \ldots, \beta_{m}\right)$ to denote a permutation of $(1, \ldots, m)$ that satisfies $\beta_{1}<\cdots<\beta_{k-l-1}$ and $\beta_{k-l}<\cdots<\beta_{m}$. Then, the CDF above is equivalent to

$$
\begin{aligned}
F_{\zeta_{l, k}}(z)= & K \frac{(l+m+1-k) !(k-l-1) !}{m !} \\
& \times \sum_{\substack{\beta_{1}<\cdots<\beta_{k-l-1} \\
\beta_{k-l}<\cdots<\beta_{m}}}\left|\left\{\begin{array}{cc}
\int_{a}^{b} \phi_{i}(y) \psi_{j}(y) \mathrm{d} y, & i=1, \ldots, n ; j=\beta_{1}, \ldots, \beta_{k-l-1} . \\
\int_{a}^{z} \phi_{i}(y) \psi_{j}(y) \mathrm{d} y, & i=1, \ldots, n ; j=\beta_{k-l}, \ldots, \beta_{m} . \\
\phi_{i, j}, & i=1, \ldots, n ; j=m+1, \ldots, n .
\end{array}\right\}\right|,
\end{aligned}
$$

with the summation over all permutations of $\boldsymbol{\beta}$, that is, $\left(\begin{array}{c}m \\ k-l-1\end{array}\right)$ in total. Substituting (B.12) into (B.6) yields the desired result.

\section{Acknowledgments}

The work of H. Zhang, X. Zhang, and D. Yang was supported by National Science and Technology Major Project of China under Grant no. 2008ZX03003-001. The work of S. Jin was supported by National Natural Science Foundation of China under Grant no. 60902009 and 60925004, and National Science and Technology Major Project of China under Grant no. 2009ZX03003-005.

\section{References}

[1] J. Wishart, "The generalised product moment distribution in samples from a normal multivariate population," Biometrika, vol. 20, no. 1-2, pp. 32-52, 1928.

[2] E. Wigner, "Characteristic vectors of bordered matrices with infinite dimensions," Annals of Mathematics, vol. 62, pp. 546564, 1955.

[3] V. A. Marčenko and L. A. Pastur, "Distributions of eigenvalues for some sets of random matrices," Mathematics of the USSRSbornik, vol. 1, pp. 457-483, 1967.

[4] A. M. Tulino and S. Verdú, Random Matrix Theory and Wireless Communications, now publishers, Boston, Mass, USA, 2004.
[5] R. K. Mallik, "The pseudo-Wishart distribution and its application to MIMO systems," IEEE Transactions on Information Theory, vol. 49, no. 10, pp. 2761-2769, 2003.

[6] I. E. Telatar, "Capacity of multi-antenna Gaussian channels," European Transactions on Telecommunications, vol. 10, no. 6, pp. 585-595, 1999.

[7] M. Chiani, M. Z. Win, and A. Zanella, "On the capacity of spatially correlated MIMO Rayleigh-fading channels," IEEE Transactions on Information Theory, vol. 49, no. 10, pp. 23632371, 2003.

[8] P. J. Smith, S. Roy, and M. Shafi, "Capacity of MIMO systems with semicorrelated flat fading," IEEE Transactions on Information Theory, vol. 49, no. 10, pp. 2781-2788, 2003.

[9] S. H. Simon, A. L. Moustakas, and L. Marinelli, "Capacity and character expansions: moment-generating function and other exact results for MIMO correlated channels," IEEE Transactions on Information Theory, vol. 52, no. 12, pp. 53365351, 2006.

[10] H. Shin, M. Z. Win, J. H. Lee, and M. Chiani, "On the capacity of doubly correlated MIMO channels," IEEE Transactions on Wireless Communications, vol. 5, no. 8, pp. 2253-2264, 2006.

[11] G. Alfano, A. Lozano, A. M. Tulino, and S. Verdú, "Mutual information and eigenvalue distribution of MIMO Ricean channels," in Proceedings of the International Symposium on Information Theory and Its Applications (ISITA '04), Parma, Italy, October 2004.

[12] T. K. Y. Lo, "Maximum ratio transmission," IEEE Transactions on Communications, vol. 47, no. 10, pp. 1458-1461, 1999.

[13] P. A. Dighe, R. K. Mallik, and S. S. Jamuar, "Analysis of transmit-receive diversity in Rayleigh fading," IEEE Transactions on Communications, vol. 51, no. 4, pp. 694-703, 2003. 
[14] S. Jin, M. R. McKay, K.-K. Wong, and X. Gao, "Transmit beamforming in Rayleigh product MIMO channels: capacity and performance analysis," IEEE Transactions on Signal Processing, vol. 56, no. 10, pp. 5204-5221, 2008.

[15] M. Kang and M.-S. Alouini, "Largest eigenvalue of complex wishart matrices and performance analysis of MIMO MRC systems," IEEE Journal on Selected Areas in Communications, vol. 21, no. 3, pp. 418-426, 2003.

[16] M. R. McKay, A. J. Grant, and I. B. Collings, "Performance analysis of MIMO-MRC in double-correlated Rayleigh environments," IEEE Transactions on Communications, vol. 55, no. 3, pp. 497-507, 2007.

[17] S. Jin, M. R. McKay, X. Gao, and I. B. Collings, "Asymptotic SER and outage probability of MIMO MRC in correlated fading," IEEE Signal Processing Letters, vol. 14, no. 1, pp. 9-12, 2007.

[18] S. Jin, M. R. Mckay, X. Gao, and I. B. Collings, "MIMO multichannel beamforming: SER and outage using new eigenvalue distributions of complex noncentral Wishart matrices," IEEE Transactions on Communications, vol. 56, no. 3, pp. 424-434, 2008.

[19] L. G. Ordóñez, D. P. Palomar, A. Pagès-Zamora, and J. R. Fonollosa, "High-SNR analytical performance of spatial multiplexing MIMO systems with CSI," IEEE Transactions on Signal Processing, vol. 55, no. 11, pp. 5447-5463, 2007.

[20] L. G. Ordóñez, D. P. Palomar, and J. R. Fonollosa, “Ordered eigenvalues of a general class of Hermitian random matrices with application to the performance analysis of MIMO systems," IEEE Transactions on Signal Processing, vol. 57, no. 2, pp. 672-689, 2009.

[21] M. Chiani and A. Zanella, "Joint distribution of an arbitrary subset of the ordered eigenvalues of wishart matrices," in Proceedings of the IEEE International Symposium on Personal, Indoor and Mobile Radio Communications (PIMRC '08), pp. 1-6, Cannes, France, September 2008.

[22] D. Gesbert, H. Bölcskei, D. A. Gore, and A. J. Paulraj, "Outdoor MIMO wireless channels: models and performance prediction," IEEE Transactions on Communications, vol. 50, no. 12, pp. 1926-1934, 2002.

[23] H. Shin and J. H. Lee, "Capacity of multiple-antenna fading channels: spatial fading correlation, double scattering, and keyhole," IEEE Transactions on Information Theory, vol. 49, no. 10, pp. 2636-2647, 2003.

[24] A. Maaref and S. Aïssa, "Impact of spatial fading correlation and keyhole on the capacity of MIMO systems with transmitter and receiver CSI," IEEE Transactions on Wireless Communications, vol. 7, no. 8, pp. 3218-3229, 2008.

[25] S. Loyka and A. Kouki, "On MIMO channel capacity, correlations, and keyholes: analysis of degenerate channels," IEEE Transactions on Communications, vol. 50, no. 12, pp. 1886$1888,2002$.

[26] A. Müller and J. Speidel, "Characterization of mutual information of spatially correlated MIMO channels with keyhole," in Proceedings of the IEEE International Conference on Communications (ICC '07), pp. 750-755, Glasgow, Scotland, June 2007.

[27] H. Shin and M. Z. Win, "MIMO diversity in the presence of double scattering," IEEE Transactions on Information Theory, vol. 54, no. 7, pp. 2976-2996, 2008.

[28] G. Levin and S. Loyka, "Multi-keyhole MIMO channels: asymptotic analysis of outage capacity," in Proceedings of the IEEE International Symposium on Information Theory (ISIT '06), pp. 1305-1309, Seattle, Wash, USA, July 2006.
[29] S. Yang and J.-C. Belfiore, "On the diversity of Rayleigh product channels," in Proceedings of the IEEE International Symposium on Information Theory (ISIT '07), pp. 1276-1280, Nice, France, June 2007.

[30] S. Yang and J.-C. Belfiore, "Diversity-multiplexing tradeoff of double-scattering MIMO channels," IEEE Transactions on Information Theory. http://arxiv.org/abs/cs/0603124.

[31] S. Jin, M. R. McKay, C. Zhong, and K.-K. Wong, "Ergodic capacity analysis of amplify-and-forward MIMO dual-hop systems," IEEE Transactions on Information Theory, vol. 56, no. 5, pp. 2204-2224, 2010.

[32] A. Firag, P. J. Smith, and M. R. McKay, "Capacity analysis for MIMO two-hop amplify-and-forward relaying systems with the source to destination link," in Proceedings of the IEEE International Conference on Communications (ICC'09), pp. 16, Dresden, Germany, June 2009.

[33] J. H. Winters, "Optimum combining in digital mobile radio with cochannel interference," IEEE Journal on Selected Areas in Communications, vol. 2, no. 4, pp. 528-539, 1984.

[34] M. Chiani, M. Z. Win, A. Zanella, R. K. Mallik, and J. H. Winters, "Bounds and approximations for optimum combining of signals in the presence of multiple cochannel interferers and thermal noise," IEEE Transactions on Communications, vol. 51, no. 2, pp. 296-307, 2003.

[35] M. Chiani, M. Z. Win, and A. Zanella, "Error probability for optimum combining of $M$-ary PSK signals in the presence of interference and noise," IEEE Transactions on Communications, vol. 51, no. 11, pp. 1949-1957, 2003.

[36] M. Chiani, M. Z. Win, and A. Zanella, "On optimum combining of $M$-PSK signals with unequal-power interferers and noise," IEEE Transactions on Communications, vol. 53, no. 1, pp. 44-47, 2005.

[37] M. R. Mckay, A. Zanella, I. B. Collings, and M. Chiani, "Error probability and SINR analysis of optimum combining in Rician fading," IEEE Transactions on Communications, vol. 57, no. 3, pp. 676-687, 2009.

[38] M. Kang and M.-S. Alouini, "Quadratic forms in complex Gaussian matrices and performance analysis of MIMO systems with cochannel interference," IEEE Transactions on Wireless Communications, vol. 3, no. 2, pp. 418-431, 2004.

[39] M. Kang, L. Yang, and M.-S. Alouini, "Outage probability of MIMO optimum combining in presence of unbalanced cochannel interferers and noise," IEEE Transactions on Wireless Communications, vol. 5, no. 7, pp. 1661-1668, 2006.

[40] S. Jin, M. R. McKay, K.-K. Wong, and X. Gao, "MIMO multichannel beamforming in interference-limited ricean fading channels," in Proceedings of the IEEE Global Telecommunications Conference (GLOBECOM '08), pp. 1012-1016, New Orleans, La, USA, December 2008.

[41] L. Sun, M. R. McKay, and S. Jin, "Analytical performance of MIMO multichannel beamforming in the presence of unequal power Cochannel Interference and Noise," IEEE Transactions on Signal Processing, vol. 57, no. 7, pp. 2721-2735, 2009.

[42] Y. Zeng and Y.-C. Liang, "Eigenvalue-based spectrum sensing algorithms for cognitive radio," IEEE Transactions on Communications, vol. 57, no. 6, pp. 1784-1793, 2009.

[43] L. S. Cardoso, M. Debbah, P. Bianchi, and J. Najim, "Cooperative spectrum sensing using random matrix theory," in Proceedings of the 3rd International Symposium on Wireless Pervasive Computing (ISWPC '08), pp. 334-338, Santorini, Greece, May 2008.

[44] F. Penna, R. Garello, D. Figlioli, and M. A. Spirito, "Exact nonasymptotic threshold for eigenvalue-based spectrum sensing," 
in Proceedings of the 4th International Conference on Cognitive Radio Oriented Wireless Networks and Communications (CROWNCOM '09), pp. 1-5, Hannonver, Vietnam, June 2009.

[45] A. Zanella, M. Chiani, and M. Z. Win, "On the marginal distribution of the eigenvalues of wishart matrices," IEEE Transactions on Communications, vol. 57, no. 4, pp. 10501060, 2009.

[46] A. Zanella and M. Chiani, "The PDF of the 1th largest eigenvalue of central wishart matrices and its application to the performance analysis of MIMO systems," in Proceedings of the IEEE Global Telecommunications Conference (GLOBECOM '08), pp. 1062-1067, New Orleans, La, USA, December 2008.

[47] H. Zhang, M. R. McKay, and D. Yang, "MIMO multi-channel beamforming in double-scattering channels," in Proceedings of the IEEE International Conference on Acoustics, Speech and Signal Processing (ICASSP '09), pp. 2093-2096, Taipei, Taiwan, April 2009.

[48] S. Kumar and A. Pandey, "Random matrix model for Nakagami-Hoyt fading," IEEE Transactions on Information Theory, vol. 56, no. 5, pp. 2360-2372, 2010.

[49] G. Alfano, A. M. Tulino, A. Lozano, and S. Verdú, "Eigenvalue statistics of finite-dimensional random matrices for MIMO wireless communications," in Proceedings of the IEEE International Conference on Communications (ICC '06), vol. 9, pp. 4125-4129, Istanbul, Turkey, June 2006.

[50] I. S. Gradshteyn and I. M. Ryzhik, Table of Integrals, Series, and Products, Academic Press, New York, NY, USA, 5th edition, 1996.

[51] C. Zhong, S. Jin, and K.-K. Wong, "Performance analysis of a Rayleigh-product MIMO channel with receiver correlation and cochannel interference," in Proceedings of the IEEE International Conference on Acoustics, Speech and Signal Processing (ICASSP '08), pp. 2877-2880, Las Vegas, Nev, USA, April 2008.

[52] C. D. Meyer, Matrix Analysis and Applied Linear Algebra, SIAM, Philadelphia, Pa, USA, 2000.

[53] P. Almers, F. Tufvesson, and A. F. Molisch, "Keyhole effect in MIMO wireless channels: measurements and theory," IEEE Transactions on Wireless Communications, vol. 5, no. 12, pp. 3596-3604, 2006.

[54] D. Chizhik, G. J. Foschini, M. J. Gans, and R. A. Valenzuela, "Keyholes, correlations, and capacities of multielement transmit and receive antennas," IEEE Transactions on Wireless Communications, vol. 1, no. 2, pp. 361-368, 2002.

[55] L. G. Ordóñez, A. Pagès-Zamora, and J. R. Fonollosa, "Diversity and multiplexing tradeoff of spatial multiplexing MIMO systems with CSI," IEEE Transactions on Information Theory, vol. 54, no. 7, pp. 2959-2975, 2008.

[56] J. G. Proakis, Digital Communications, McGraw-Hill, New York, NY, USA, 4th edition, 2001.

[57] L. G. Ordóñez, D. P. Palomar, A. Pagès-Zamora, and J. R. Fonollosa, "Minimum BER linear MIMO transceivers with adaptive number of substreams," IEEE Transactions on Signal Processing, vol. 57, no. 6, pp. 2336-2353, 2009.

[58] A. Maaref and S. Aïssa, "Eigenvalue distributions of wisharttype random matrices with application to the performance analysis of MIMO MRC systems," IEEE Transactions on Wireless Communications, vol. 6, no. 7, pp. 2678-2689, 2007.

[59] A. Maaref and S. Aïssa, "Joint and marginal eigenvalue distributions of (Non)central complex wishart matrices and PDF-based approach for characterizing the capacity statistics of MIMO ricean and rayleigh fading channels," IEEE Transactions on Wireless Communications, vol. 6, no. 10, pp. $3607-$ 3619, 2007.
[60] H. A. David and H. N. Nagaraja, Order Statistics, John Wiley \& Sons, Hoboken, NJ, USA, 3rd edition, 2003. 